\title{
41. APORTACIONES AL CONOCIMIENTO DE LA FLORA DEL ALTO VALLE DEL GUADALQUIVIR (JAÉN, S España)
}

\author{
Antonio GARCÍA FUENTES, Manuel MELENDO LUQUE, \\ Néstor SÁNCHEZ PASCUAL y Eusebio CANO CARMONA
}

Contributions to the knowledge of the flora in Guadalquivir High Valley (Jaen, S Spain)

Palabras clave. Flora, Corología, Fitosociología, Jaén, España.

Key words. Flora, Chorology, Phytosociology, Jaen, Spain.

Habiendo realizado un estudio de la vegetación y flora en el territorio conocido como alto valle del Guadalquivir (Jaén, España), se presentan aquí una serie de taxones de especial interés por ser nuevas citas para la provincia de Jaén, o primeras citas para Andalucía oriental; asímismo, se hace una ampliación de areal corológico de algunos taxones. Todos los pliegos mencionados se hallan depositados en el Herbario JAEN de la Facultad de Ciencias Experimentales de la Universidad de Jaén.

\section{Atriplex tornabenei Tineo ex Guss.}

JAÉN: Fuerte del Rey, 30SVG1896, 330m, 13/07/93, García Fuentes \& Cano, JAEN 96728.

Propio de zonas ricas en sales y con cierta nitrificación. Aparece sobre los materiales triásicos del Keuper presentes en la zona meridional del alto valle, en zonas de vaguada donde por escorrentía se acumulan las aguas que lavan los materiales gípsicos.

\section{Spergularia tangerina $\mathrm{P}$. Monnier}

JAÉN: Arroyo de la Cuevezuela, 30SVG3686, 400m, 31/05/95, A. García Fuentes, JAEN 96752.

Elemento íbero-norteafricano propio de zonas halófilas. Presente en comunidades de Parapholi-Frankenietum pulverulentae subas. spergularietosum tangerinae. Es primera cita para Andalucía oriental (Ratter, 1990:155).
Lavatera maroccana (Batt. \& Trabut) Maire JAÉN: Marmolejo, Loma de la Marquesa, 30SUH9210, 240m, 16/05/91, A. García Fuentes \& E. Cano, JAEN 96751.

Es abundante en escombreras, basureros y lugares altamente nitrificados. En comunidades de la asociación Lavateretum arboreo-creticae. Propia del SW de España y NW de África, es primera cita para Andalucía oriental.

Malope trifida Cav.

JAÉN: Cementerio Viejo, 30SVG3181, 300m, 14/04/93, A. García Fuentes \& E. Cano, JAEN 96737.

En los herbazales ruderalizados de Ruderali-Secalietea cerealis.

\section{Helianthemum almeriense $\mathrm{Pau}$}

JAÉN: Ctra. Jaén-Fuerte del Rey, Km. 6, 30SVG2687, 350m, 9/04/92, A. García Fuentes \& E. Cano, JAEN 96735.

Endemismo del SE árido de la Península Ibérica, presente también en Marruecos (Castroviejo et al., 1993), que llega a la provincia de Jaén a través de las zonas Guadiciano-Baztetanas. Es un elemento propio de tomillares y lugares despejados, en suelos secos, margosos y con abundante yeso.

Helianthemum apenninum (L.) Mill. subsp. stoechadifolium (Brot.) Samp.

JAÉN: Proximidades a Puente Tablas, 30SVG3484, 600m, 14/04/93, A. García 
Fuentes, JAEN 96736.

Este taxón iberonorteafricano no se ha citado para la provincia de Jaén (López González, 1993:382). Detectado en zonas margosas y margo-yesíferas, siempre sobre suelos muy pobres y compactados.

\section{Tamarix parviflora DC.}

JAÉN: Sorihuela de Guadalimar, Río Guadalimar, 30SVH9729, 550m, 17/05/94, A. García Fuentes, JAEN 96742.

En zonas húmedas y arroyos no salobres. Según Flora Ibérica (Cirujano, 1993) no existe cita provincial para este taxón. Se presenta en comunidades de la clase Nerio-Tamaricetea.

Salix x multidentata T.E. Díaz \& Llamas

JAÉN: Villanueva del Arzobispo, Arroyo los Olmillos, 30SVH9827, 600m, 17/05/94, A. García Fuentes, JAEN 96753.

No hay referencias para la provincia de Jaén de este sauce, recientemente descrito (Díaz \& Llamas, 1987:134) que se ha detectado sobre materiales triásicos asentados sobre areniscas rojas descarbonatadas.

\section{Hedysarum coronarium L.}

JAÉN: Proximidades Campus Universitario, 30SVG3181, 510m, 3/06/93, A. García Fuentes, JAEN 96733.

Taxón poco frecuente que se desarrolla sobre suelos margosos. En pastizales subnitrófilos de Sisymbrietalia officinalis.

Hedysarum glomeratum F. G. Dietrich

JAÉN: Fuerte del Rey. Ayo. Las Cadenas, 30SVG1996, 400m, 7/05/92, A. García Fuentes \& E. Cano, JAEN 96734.

Planta que se desarrolla sobre margas calizas del alto valle del Guadalquivir, en herbazales subnitrófilos de Bromenalia rubenti-tectori.

Ononis viscosa L. subsp. subcordata (Cav.) Sirj. JAÉN: Ctra. Jaén-Torrequebradilla, Km.
6, 30SVG3686, 650m, 5/06/92, A. García Fuentes \& E. Cano, JAEN 96738.

Se desarrolla en substratos básicos y herbazales subnitrófilos pertenecientes al suborden Bromenalia rubenti-tectori.

Ornithopus sativus Brot. subsp. isthmocarpus (Cosson) Dostál.

JAÉN: Marmolejo, Río Yeguas, 30SUH9113, 200m, 5/04/95, A. García Fuentes, JAEN 96739.

En prados húmedos sobre suelos arenosos. Es un taxón que en la Península solo aparece en las provincias Luso-Extremadurense, Gaditano-Onubo-Algarviense y Bética. En pastizales de Tuberarietea guttatae.

Vicia villosa Roth subsp. eriocarpa (Hausskn.) P.W. Ball

JAÉN: Andújar, Arroyo Escobar, 30SVH1511, 250m, 8/04/93, A. García Fuentes, JAEN 96743.

En comunidades subnitrófilas de Taeniathero-Aegilopion geniculatae.

\section{Euphorbia lagascae Sprengel}

JAÉN: Ctra. Jaén-Fuerte del Rey, 30SVG2787, 550m, 7/05/92, A. García Fuentes \& E. Cano, JAEN 96730.

Taxón citado en diferentes provincias andaluzas (Sevilla, Córdoba y Almería). Se desarrolla sobre suelos margosos de la campiña con cierto contenido en arcillas.

Euphorbia medicaginea Boiss.

JAÉN: Marmolejo. Loma de la Marquesa, 30SUH9410, 220m, 21/04/91, A. García Fuentes \& E. Cano, JAEN 96731.

Especie que se desarrolla sobre suelos básicos de la campiña. En herbazales de Ruderali-Secalietea cerealis.

Blackstonia perfoliata (L.) Hudson subsp. serotina (Roch ex Reichenb.) Vollmann JAÉN: Mengíbar, 30SVH2901, 300m, 17 / 
07/95, A. García Fuentes, JAEN 96729.

Márgenes de arroyos y zonas húmedas, en comunidades de herbazales húmedos y nitrificados dentro de la clase MolinioArrhenatheretea.

\section{Lavandula multifida $\mathrm{L}$.}

JAÉN: Marmolejo. Loma de la Marquesa, 30SUH9210, 220m, 20/04/92, A. García Fuentes \& E. Cano, JAEN 96732.

Escasa en el territorio, tan solo presente en los suelos rojos descarbonatados del Trías (Buntsandstein), siempre en el piso termomediterráneo superior. Ha sido citada, en Andalucía, en las provincias de Málaga, Almería, Cádiz, Córdoba y Granada (SuárezCervera \& Seoane-Camba, 1986).

Acanthus mollis L. subsp. platyphyllos Murb.

JAÉN: Marmolejo. Loma de la Marquesa, 30SUH9310, 230m, 16/05/91, A. García Fuentes \& E. Cano, JAEN 96727.

Planta nitrófila de relativa frecuencia en los herbazales húmedos próximos a arroyos (Molinio-Arrhenatheretea).

\section{Potamogeton fluitans Roth.}

JAÉN: Santisteban del Puerto, Río Guadalimar (Puente de Cerro Molino), 30SVH8623, 490m, 7/06/95, A. García Fuentes \& F. Ortega, JAEN 96740.

Taxón propio de arroyos y ríos de aguas dulces. Característico de la clase Potametea.

\section{Potamogeton pectinatus L.}

JAÉN: Santisteban del Puerto, Río Guadalimar (Puente de Cerro Molino), 30SVH8623, 490m, 7/06/95, A. García Fuentes \& F. Ortega, JAEN 96741.

Taxón que apetece de aguas dulces, corrientes o no, y también soporta aguas más o menos salobres. Propio de la alianza Magnopotamion.

\section{Limodorum abortivum (L.) Swartz}

JAÉN: Marmolejo, Loma de la Marquesa,
30SUH9510, 240m, 20/03/92, A. García Fuentes \& E. Cano, JAEN 96747.

Taxón que aparece en el dominio del encinar silícola y calcícola. A pesar de su relativa frecuencia, es un taxón poco citado. Se amplía su areal corológico al subsector Hispalense.

\section{BIBLIOGRAFÍA}

CAStroviejo, S. - 1986/93- In. Castroviejo et al. (eds.) Flora Ibérica. Vols. I-IV. Servicio de Publicaciones del C.S.I.C. Madrid.

CIRUJANO, S. -1993- Tamarix L. in Castroviejo, S.; Aedo, C.; Cirujano, S.; Laínz, M.; Montserrat, P.; Morales, R.; Muñoz Garmendia, F.; Navarro, C.; Paiva, J. \& Soriano, C. (eds.). Flora Ibérica. Real Jardín Botánico (C.S.I.C.), III: 730 pp. Madrid.

DÍAZ, T.E. y F. LLAMAS -1987- Aportaciones al conocimiento del género Salix L. (Salicaceae) en la provincia de León (NW España). Acta Bot. Malacitana, 12:111-150.

LÓPEZ GONZÁLEZ, G. -1993- Helianthemum Mill. In Castroviejo, S., C. Aedo, S. Cirujano, M. Laínz, P. Montserrat, R. Morales, F. Muñoz Garmendia, C. Navarro, J. Paiva \& C. Soriano (eds.). Flora Ibérica. Real Jardín Botánico (C.S.I.C.), III: 730 pp. Madrid.

RATTER, J.A. - 1990- Spergularia (Pers.) J. Presl \& K. Presl In Castroviejo, S., M. Laínz, G. López González, P. Montserrat, F. Muñoz Garmendia, J. Paiva \& L. Villar (eds.). Flora Ibérica. Real Jardín Botánico (C.S.I.C.), II: 897 pp. Madrid. SUÁREZ-CER VERA, M. y J. SEOANE-CAMBA 1986- Sobre la distribución corológica del género Lavandula L. en la Península Ibérica. Lazaroa, 9:201-220.

Aceptado para su publicación en Junio de 1996

Dirección de los autores. A. García Fuentes, M. Melendo Luque y E. Cano Carmona: Dpto. de Biología Animal, Vegetal y Ecología. Facultad de CC. Experimentales. Universidad de Jaén. 23.071-Jaén. N. Sánchez Pascual: Insto. Bachillerato Martín Alhaja. La Carolina-Jaén. 